\title{
Using Volume Guarantee (VG) to Achieve Ventilatory Efficiency in High-Frequency Oscillation (HFO)
}

Rob Graham, R.R.T./N.R.C.P.

I dedicate this column to the late Dr. Andrew (Andy) Shennan, the founder of the perinatal program at Women's College Hospital (now at Sunnybrook Health Sciences Centre). To my teacher, my mentor and the man I owe my career as it is to, thank you. You have earned your place where there are no hospitals and no NICUs, where all the babies do is laugh and giggle and sleep.

Efficiency is the relationship between how much energy goes into a system and how much work comes out of it. Peak efficiency is the point at which maximum output is achieved with the least amount of energy consumed per unit. We usually think about efficiency when talking about electrical items like appliances, air conditioners, and so forth, or a vehicle's fuel economy, but the same concept applies to ventilation.

Optimum compliance is the point at which the lungs accept the most volume using the lowest ventilating pressure. As functional residual capacity decreases or increases compliance decreases as the lungs are no longer at the top of the compliance curve, more pressure must now be used to deliver the same volume. All modes of ventilation produce better results when optimum compliance is reached. As well, the lung is most protected when being ventilated at or near optimum compliance. Clinically we refer to this as the "open lung" approach. The infamous "HIFI" trial of the 1980s demonstrated the importance of this approach during HFO.

\author{
"With HFO, there is more to efficiency \\ than compliance; regardless of \\ compliance, there is a point at which \\ settings can be adjusted to use less \\ pressure (amplitude) to provide \\ sufficient minute ventilation."
}

With HFO, there is more to efficiency than compliance; regardless of compliance, there is a point at which settings can be adjusted to use less pressure (amplitude) to provide sufficient minute ventilation. Frequency (f) plays a large part here. Due to the fixed inspiratory to expiratory (l:E) ratio, increasing frequency gives less inspiratory time to get volume in, and less expiratory time to get it out. This may lead to gas trapping in and of itself, but since the only way to maintain volume when raising frequency is to use higher amplitude, the risk of creating pinch points also increases. Because amplitude is above and below the set mean airway pressure (MAP), higher amplitudes create larger troughs during the expiratory phase as well as higher peak pressure on inspiration. As end-expiratory pressure approaches critical closing pressures,

- PEEP/Paw and the oscillatory pressure waveform must be raised to overcome gas trapping

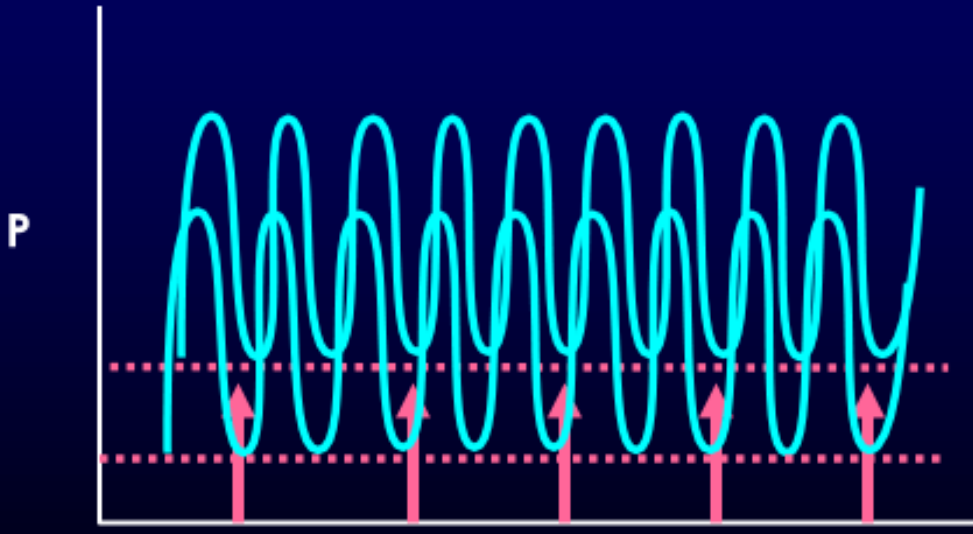

time

Figure 1: Oscillatory waveform above and below MAP. "PEEP" is the lowest point in the waveform (2) 
- Back pressure (High PEEP/Paw) may splint open the airway and allow gas to exit

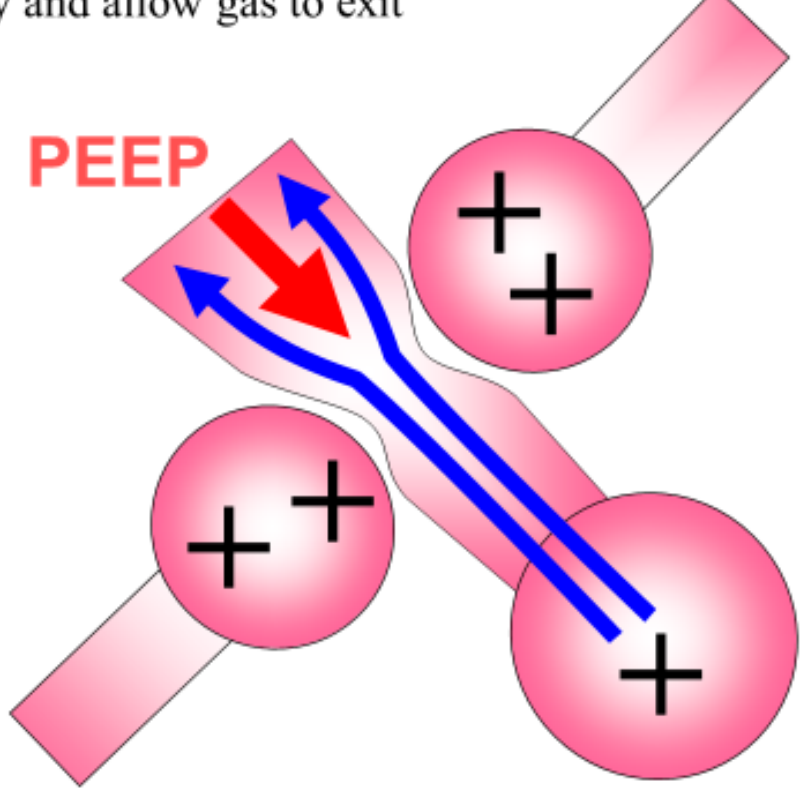

Figure 2: Pinch points occur when extraluminal pressure exceeds airway pressure during exhalation in HFO (2)

airway instability and micro-atelectasis may result. If expiratory pressure is low enough, "pinch points" occur in the conducting airways, preventing gas from exiting the lung.

Oscillators used in the U.S. to date pose a conundrum for clinicians since they do not provide enough information at the bedside. Amplitude is adjusted as "power," and while the amplitude is expressed as the pressure in $\mathrm{cmH}_{2} \mathrm{O}$, no such measurement is available, and "chest wiggle" is the basis for amplitude adjustment. When dealing with smaller babies, there is another problem: the amplitude on these first-generation machines is too high at lower frequencies to be used safely. The workaround has been to use higher frequencies, but as time constants get longer, using higher frequency greatly increases the risk of gas trapping. As well, the higher amplitude necessitated with higher frequency may

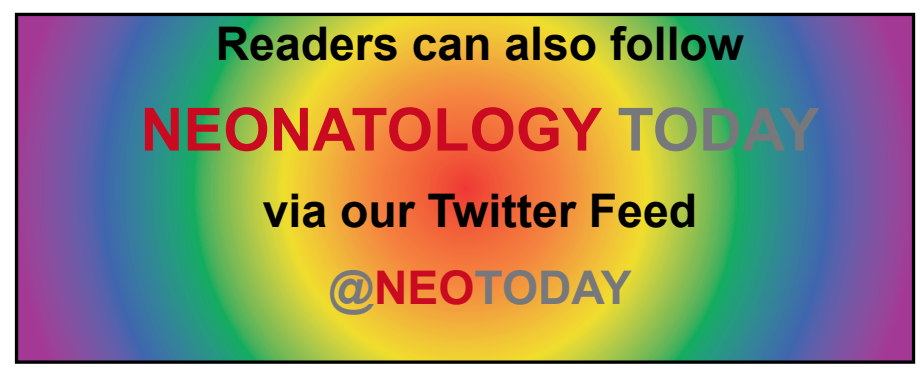

result in alterations to flow characteristics, i.e., creating turbulent flow, which reduces efficiency.

"The workaround has been to use higher frequencies, but as time constants get longer, using higher frequency greatly increases the risk of gas trapping. As well, the higher amplitude necessitated with higher frequency may result in alterations to flow characteristics, i..e., creating turbulent flow, which reduces efficiency."

The equation for $\mathrm{CO}_{2}$ clearance during $\mathrm{HFO}$ is expressed as "DCO${ }_{2}$. $\mathrm{DCO}_{2}=\mathrm{f}^{\star} \mathrm{Vt}{ }^{2}$ where $\mathrm{Vt}$ is HFO tidal volume. (2) In practice, this means that changes in frequency linearly alter $\mathrm{CO}_{2}$ while

NEONATOLOGY TODAY is interested in publishing manuscripts from Neonatologists, Fellows, NNPs and those involved in caring for neonates on case studies, research results, hospital news, meeting announcements, and other pertinent topics.

Please submit your manuscript to: LomaLindaPublishingCompany@gmail.com 
changes in volume increase $\mathrm{CO}_{2}$ exponentially. This is particularly true when using frequencies above $5 \mathrm{~Hz}$. (2) (This is an excellent primer by Dr. Jane Pillow courtesy of Drager). Clinically this means decreases in frequency can be compensated for by a small increase in Vt. In order to do that, however, it is helpful to know what that volume is. This is where VG comes into play.

Third generation oscillators soon to be available in the U.S. provide measurements for both $\mathrm{Vt}$ and minute volume and provide the option to set a target volume in HFO mode. Chest wiggle is no longer the indicator of how much ventilation a machine is providing since these machines not only tell us; they allow us to adjust the volume. In reality, what is altered to achieve a set target volume is amplitude.

Where does efficiency come into play? When decreasing frequency, we can now compensate for lost volume by simply increasing the target volume; adjustments are made in increments as small as $0.1 \mathrm{ml}$. Typical Vt during HFO is usually $1-2 \mathrm{ml} / \mathrm{kg}$, and while higher volumes may be used, it stands to reason that using higher volumes during HFO may decrease the lung protectiveness of the mode. It is my practice not to exceed $3 \mathrm{ml} / \mathrm{kg}$ when using HFO with VG unless the amplitude necessary to deliver the volume is relatively low, or the larger volume is not required for a prolonged length of time.

In clinical practice, decreasing frequency while increasing VG may result in lower amplitude being used, sometimes as low as 5 $\mathrm{cmH}_{2} \mathrm{O}$. This demonstrates increased efficiency and reduces the risk of gas trapping in two ways: lower amplitude decreases the risk of creating pinch points, and lower frequency gives more time for gas to escape. This, arguably, is a good thing. It is worth noting that while increasing frequency using VG increases $\mathrm{CO}_{2}$ elimination linearly, this is only true if the increased frequency does not result in gas trapping and that the machine is able to provide enough amplitude to do so. Otherwise, $\mathrm{CO}_{2}$ clearance may actually be impaired.

Resonance frequency also plays a role in $\mathrm{CO}_{2}$ clearance. Ventilating with frequencies near pulmonary resonant frequency increases ventilatory efficiency. Resonance frequency decreases with increasing compliance (3) and varies depending on pathology and size. Rates used during high-frequency jet ventilation are closer to resonant frequency (4) than those typically used during $\mathrm{HFO}$ and are generally less than $10 \mathrm{~Hz}$. Increasing frequency during HFO

\section{"It is worth noting that while increasing frequency using $V G$ increases $\mathrm{CO}_{2}$ elimination linearly, this is only true if the increased frequency does not result in gas trapping and that the machine is able to provide enough amplitude to do so. Otherwise, $\mathrm{CO}_{2}$ clearance may actually be impaired."}

may reduce any benefit derived from the resonant frequency.

Another benefit of the greater monitoring capability of these machines is the ability to monitor compliance in real-time. If an adjustment in MAP results in the machine using more amplitude to maintain volume, this reflects decreased compliance and tells us the move is not a wise one. This is true of other modes of ventilation as well: if adjusting PEEP in a conventional mode or highfrequency jet ventilation necessitates using higher pressure to achieve the same results, the message is the same. An increase in oxygen requirements is another indicator, but derecruitment may show up as higher ventilating pressure before oxygen requirements change significantly. If the maximum amplitude is adjusted fairly closely to amplitude used, a clinician also is given an early warning of decreasing compliance when the machine gives an alarm that it cannot deliver set volume.

While HFO/VG represents a brand-new concept in HFO, it is important to note that this mode has been used outside the U.S. safely and with success, and the adjunct is now available from several manufacturers, including Drager, Lowenstein Medical, and Maquet. As with any new modality, buy-in from clinicians has been mixed. In Canada, there seems to be a great divide; those west of Ontario appear not to use the adjunct frequently, if at all. Units in Toronto have embraced HFO/VG, and it is used extensively here, especially in the unit where I practice.

Finally, allow me to wish everyone a good summer. Stay healthy and stay safe during these trying times. As our mothers have told us, "this too shall pass."

\section{References:}

$1 \quad$ Figures 1 and 2 courtesy of Bunnell Inc.

2 https://www.draeger.com/Library/Content/hfov-bk-9102693en.pdf

3 https://link.springer.com/article/10.1007/BF02072632

4 https://onlinelibrary.wiley.com/doi/pdf/10.1111/j.1399-6576.1989. tb03006.x

Disclosures: The author receives compensation from Bunnell Inc for teaching and training users of the LifePulse HFJV in Canada. $\mathrm{He}$ is not involved in sales or marketing of the device nor does he receive more than per diem compensation. Also, while the author practices within Sunnybrook H.S.C. this paper should not be construed as Sunnybrook policy per se. This article contains elements considered "off label" as well as maneuvers, which may sometimes be very effective but come with inherent risks. As with any therapy, the risk-benefit ratio must be carefully considered before they are initiated.

NT

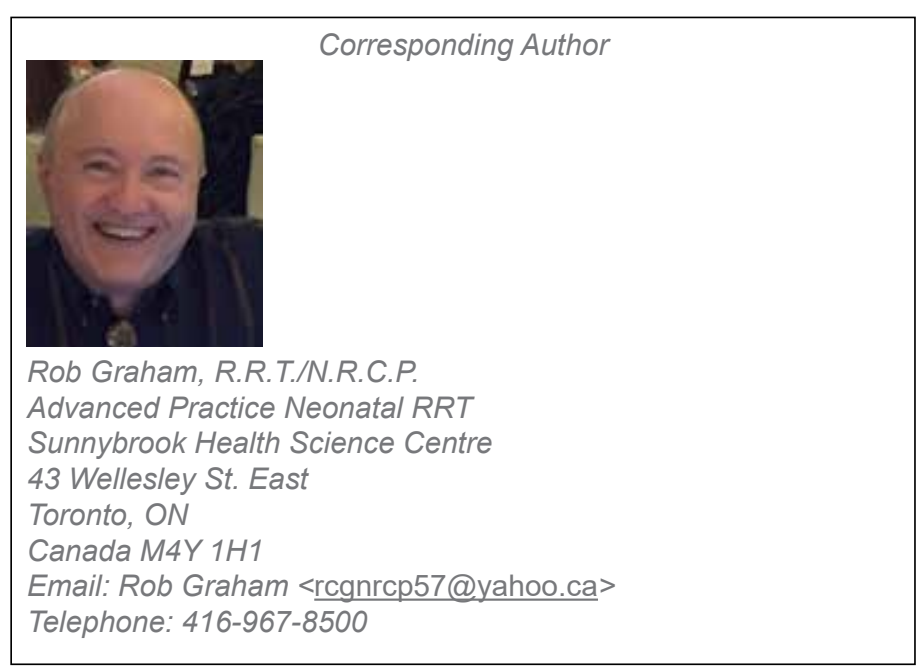

